\title{
Repurposing drugs to treat disturbances of learning and memory in neuropsychiatric disorders
}

\author{
Dr Sunjeev Kamboj and Dr Ravi Das
}

Citation: Clinical Pharmacist, March 2018, Vol 10, No 3, online | DOI: 10.1211/CP.2018.20204477

In high-income countries, such as the UK, mental health problems are among the most prevalent medical conditions. These conditions can have a devastating impact on the population; for example, accidental poisoning from opioid addiction is one of the leading causes of death among younger adults in the United States (US). While the UK has not faced a similar opioid epidemic, addiction and other psychiatric disorders still contribute to our public health burden (Mordecai et al., 2018).

Despite the pressing need for new and more effective treatments, many pharmaceutical companies have moved away from researching psychiatric medicines. A string of high-profile failed phase III trials may further discourage investment in psychiatric drug development. There are effective alternatives to psychiatric medications - talking therapies, for example - but their outcomes are generally no better than treatment with drugs. And around half of patients with anxiety and depression fail to recover by the end of treatment.

The basic neurobiological and psychological processes that underlie psychiatric disorders are only beginning to be uncovered. The disorders are seen as a 'moving target': many distinct symptoms manifest differently in different people.

There has been historical (and continued) over-reliance on pathognomonic (characteristic) diagnostic frameworks, such as the American Psychiatric Association's Diagnostic and Statistical Manual of Mental Disorders. A recent drive by the National Institute of Mental Health in the United States - the Research Domain Criteria initiative — has encouraged a better understanding of the fundamental psychological and biological processes that are common across psychiatric disorders.

When psychiatric symptoms are viewed 'transdiagnostically' (ie. by focusing on symptoms rather than on a diagnosis), treatment of a wide array of distinct symptoms can become more manageable because only a small set of disordered processes must be targeted. With a focus on common processes, the overlapping causes and maintaining factors of distinct disorders also become clearer.

Psychiatric disorders often occur concurrently, so reversing a limited number of disordered psychological processes may have an impact on a range of co-morbid problems. Among these processes, a dysregulation of learning and memory seems to be critical in the onset and maintenance of anxiety disorders and addiction.

At first sight, these disorders do not have much in common. Addiction, for example, involves a loss of control over drug taking, whereas people with anxiety disorders often have 
difficulties associated with 'over-control' (of feelings and behaviours). Yet, there are indeed similarities in the psychological processes involved in both disorder types, especially in relation to the role of learning and memory. Consider, for example, patients with severe alcohol problems. Obtaining and consuming alcohol is an overriding priority for them, and these behaviours often occur at the expense of other important activities. Thoughts about alcohol and drinking come to mind automatically, evoked by reminders of alcohol-use in the environment. For example, the smell or sight of alcohol readily triggers intense craving, followed by excessive drinking, despite attempts to resist. In alcohol addiction, these behavioural 'routines' are so well-rehearsed and stereotyped that they can be triggered relatively automatically, promoting drinking without deliberation ('mindless' drinking). In one way or another, all of these phenomena (intrusive thoughts about alcohol; craving; drinking without thinking) reflect abnormal learned responses stored in 'maladaptive memory traces.'

Similarly, in phobias and posttraumatic stress disorder (PTSD), physiological, behavioural and cognitive responses are triggered when the individual is confronted by the feared situation or object, reflecting learned associations between a feared stimulus and a fear response. Moreover, visual memories, in the form of distressing mental images of frightening situations or phobic objects come to mind unintentionally (e.g. in the form of 'flashbacks'). Again then, disordered learning and memory play a core role in fear-related disorders.

Learning is the process of forming new associations between occurrences in the outside world and events inside the body. Usually these co-occurrences need to happen repeatedly to cement the associations into long-term memory. For example, repeated drug-taking in a particular environment, leads to the formation of a 'drug memory,' which is automatically activated the next time the person is in that environment, resulting in craving and reflexive drug-seeking and consumption. But repetition isn't always necessary for strong learning to occur. Some events are so salient or stressful that a single experience can leave an indelible mark on the mind. For example, traumatic memories are formed after a single life-threatening event (like a car accident), and activation of the traumatic memory (e.g. by the sound of a speeding car) results in fear, and the urge to escape (resulting in, for example, avoidance of busy roads).

At the cellular level, learning involves the formation and sculpting of synaptic connections between neurons in key areas of the brain (like the amygdala and hippocampus), whereas 'remembering' involves reactivation of the networks of neural connections formed during learning. Much contemporary research in neuroscience has focused on the cellular and molecular mechanisms underlying these neural processes. Behavioural neuroscientists often use rodent models of psychiatric disorders to tease apart the role of specific brain circuits in behavioural dysregulation. They employ a variety of techniques, including localised pharmacological treatments directed at specific brain regions, to decipher the role of particular neurotransmitter systems in those regions. These studies have shown that abnormal neurotransmission is associated with dysregulation in fear and reward learning circuitry in rodent models of anxiety and addiction respectively. Modern neuroimaging techniques show similar neural changes in the equivalent brain structures in people with anxiety and addiction.

In our research at the Clinical Psychopharmacology Unit at UCL, we use insights from laboratory studies in animals to study how learning and memory processes go awry, and to 
understand how these processes might be ameliorated in humans. We do this by, for example, training participants to be afraid of simple auditory and visual stimuli by repeatedly pairing these with uncomfortable electric shocks ('Pavlovian conditioning'). After a number of repetitions, participants begin to respond fearfully to the harmless auditory/visual stimuli, a simulation of the processes that occur naturally in specific phobias. In other experiments, we show participants disturbing film scenes, causing them to remember these scenes involuntarily over the next several days (equivalent to flashbacks in PTSD). By simulating phobic and traumatic reactions, we have been able to ask whether the symptoms of phobias and PTSD can be reversed using drugs that interfere with the neural processes involved in laying down fearful or traumatic memories. If so, these drugs could be developed to prevent or treat these disorders.

Drugs like propranolol and hydrocortisone, which have well known anti-hypertensive and anti-inflammatory properties respectively, can also impair certain types of learning and memory. This might initially seem surprising, since acute cognitive impairment is not a commonly reported side effect of these drugs, at least in people without systemic diseases. In fact, their memory impairing effects only seem to occur under specific and time-limited circumstances. In particular, amnesia for certain types of (emotional) information is evident if propranolol or hydrocortisone are administered within a brief window of opportunity, when the memory has not yet fully stabilised or 'consolidated'. Memory consolidation - a process that unfolds over several hours after initial learning - is driven by intense synaptic protein synthesis and post-translational modification, leading to the remodelling of synapses and a 'cementing' of memories in long-term storage. $\beta$-adrenoceptor and corticosteroid antagonists are thought to prevent these structural and functional changes at synapses in brain regions involved in processing emotional information, and as a result, these drugs prevent the formation of long-term emotional memories.

These discoveries were originally made in non-human animals using direct infusions of propranolol and corticosteroids into brain regions critical for emotional learning and memory (e.g. the amygdala and hippocampus; McGaugh, 2004). However, oral propranolol and hydrocortisone, which are lipophilic and readily cross the blood-brain barrier, can also impair emotional memory formation in humans. Early studies investigated memory for simple stimuli (lists of words and pictures), and showed that the effects of these drugs were quite circumscribed: they appeared to strip away the negative emotions associated recalling unpleasant events (e.g. Cahill et al, 1994). These basic psychopharmacological experiments provided a rationale for testing the effects of propranolol and hydrocortisone in clinical trials targeting much more distressing memories, like those seen in PTSD. The results have been promising (especially with hydrocortisone; Sijbrandij et al, 2015). More importantly perhaps, these trials are an excellent example of how neuroscience-driven research can lead to a repurposing of drugs for novel applications in psychiatry. Such re-purposing can overcome the enormous financial barriers and high failure rates associated with bringing new compounds to market.

In our own experiments, we have tested whether other drugs might also interfere with pathological learning and memory in PTSD and addictions, focusing primarily on glutamatergic antagonists. Since glutamatergic signalling, particularly at NMDA receptors, is fundamental to synaptic plasticity (and therefore memory consolidation), its disruption may provide an additional route to preventing the long-term storage of fear- or addiction-related 
memories. We showed, for example, that compared to medical air, the anaesthetic gas and NMDA antagonist, nitrous oxide, can speed up the reduction in distressing intrusive memories in the days after a simulated traumatic event (Das et al, 2016). These findings provided preliminary evidence that nitrous oxide, a widely used drug with an excellent safety profile, might also be useful in preventing the onset of PTSD. Our ongoing research with nitrous oxide also suggests that it may have similar effects in weakening addiction-related memories.

In particular, our experiments on addiction-related memories assess whether it is possible to interfere with the pre-existing memories that drive heavy drinking. In these studies, participants' associations between alcohol and reward are already well-established (they are formed through repeatedly pairing alcohol-related stimuli or 'cues' with the intoxicating and rewarding effects of alcohol in participants' natural environments). These associations are likely to be very strong, as they are formed over years of drinking. A key question remains whether it is possible to interfere with pre-existing memories that are strongly (and naturally) 'trained'. To address this, our translational approach is currently focussing on a very promising memory plasticity process called memory reconsolidation (Kamboj \& Das, 2017). This process involves the 'updating' of consolidated memories, and is triggered when these memories are retrieved in the presence of new, relevant information. This updating requires a brief period of memory 'destabilisation' which provides an additional window of opportunity (similar to that afforded when the memory is initially being consolidated) to use the aforementioned amnestic drugs to weaken pre-existing memories in addiction or anxiety disorders. Our preliminary findings with glutamatergic antagonists, suggest that this is a promising approach in heavy drinkers.

We believe these memory-weakening approaches have enormous potential in psychiatric treatment. However, they are only concerned with processes occurring within the individual and we should not lose sight of the role that social factors play in psychiatric disorders. The opioid crisis in the US challenges our assumptions about what it means to be 'an addict', as individuals across the age and socioeconomic spectrum are affected by opioid addiction. Here in the UK, researchers have found a link between socioeconomic deprivation and increased rates of opioid prescription (Mordecai et al., 2018). This highlights that social and economic distress play an extremely important role in the development and maintenance of psychiatric disorder. Any isolated intervention approach, for example, one that only targets abnormal psychological processes within the individual, while ignoring critical environmental contributors to distress, is unlikely to be effective in the long term. Once we've established the therapeutic potential of memory interfering pharmacological strategies, the next step will be to integrate these into holistic treatments that provide comprehensive support to people with anxiety and substance use disorders.

\section{References}

Cahill, L, Prins B, Weber M, \& McGaugh JL (1994) Beta-adrenergic activation and memory for emotional events. Nature, 371(6499), 702-704

Das, R. K., Tamman, A., Nikolova, V., Freeman, T. P., Bisby, J. A., Lazzarino, A. I., \& Kamboj, S. K. (2016). Nitrous oxide speeds the reduction of distressing intrusive memories 
in an experimental model of psychological trauma. Psychological Medicine, 46(8), 17491759.

Kamboj SK, Das RK (2017). Behavioral and Pharmacological Strategies for Weakening Maladaptive Reward Memories: A New Approach to Treating a Core Disease Mechanism in Tobacco Use Disorder. JAMA Psychiatry, 74(3):209-211.

McGaugh, J. L. (2004). The amygdala modulates the consolidation of memories of emotionally arousing experiences. Annual Review of Neuroscience, 27, 1-28.

Mordecai, L., Reynolds, C., Donaldson, L. J., \& de C Williams, A. C. (2018). Patterns of regional variation of opioid prescribing in primary care in England: a retrospective observational study. British Journal of General Practice, bjgp18X695057.

Sijbrandij, M., Kleiboer, A., Bisson, J. I., Barbui, C., \& Cuijpers, P. (2015). Pharmacological prevention of post-traumatic stress disorder and acute stress disorder: a systematic review and meta-analysis. The Lancet Psychiatry, 2(5), 413-421. 